\title{
NONCOMPACT UNIMODULAR GROUPS WITH PURELY ATOMIC PLANCHEREL MEASURES
}

\author{
GIANCARLO MAUCERI AND MASSIMO A. PICARDELLO
}

\begin{abstract}
A noncompact unimodular group whose regular representation decomposes as a direct sum of irreducible subrepresentations was constructed by J. M. G. Fell. In the present paper, groups of this kind are studied, and several new examples are provided to settle open structure problems.
\end{abstract}

1. Introduction. The existence of a noncompact unimodular group whose regular representation decomposes into a direct sum of irreducible constituents was proved in [1] by means of an example due to J. M. G. Fell. This example consists of the semidirect product $G_{1}=\mathcal{T}_{p} \cdot \mathscr{2}_{p}$ of the group $\mathcal{T}_{p}$ of all $p$-adic numbers of valuation 1 and of the additive group of the $p$-adic field $\mathcal{Q}_{p}$, where $\mathcal{T}_{p}$ acts on $\mathcal{Q}_{p}$ by multiplication. Groups with the aforementioned property, called "Fell groups", were studied in [7], where it was shown that the formal degrees of their square-integrable representations are not bounded away from zero (this is also a direct consequence of the fact that the $L^{2}$-conjecture is true, in other words, that $L^{2}(G)$ is not a convolution algebra unless $G$ is compact; recall that the formal degree of a square-integrable irreducible representation $\pi$ is the Fourier algebra norm of the minimal idempotents belonging to the minimal translation biinvariant subspace of $L^{2}(G)$ corresponding to $\pi$ : see [7], [8] for terminology and details). The aim of this paper is to produce several new examples of Fell groups: most of these examples are obtained by generalizing Fell's construction in several different ways, and, as one would expect, they turn out to be amenable $p$-adic matrix groups; however, we will also exhibit nonamenable $p$-adic Fell groups and non-p-adic Fell groups.

In $\$ 2$ we construct, for every integer $n$, a Fell group of $p$-adic matrices of dimension $n$ which coincides with Fell's example for $n=1$ and is not solvable for $n>1$; these groups can be regarded in a natural sense as the groups of "rigid motions" of $2_{p}^{n}$.

As we noted in [7], the quotient of a Fell group $G$ by a compact normal subgroup is again a Fell group. This is so since the quotient is unimodular and noncompact, and its regular representation lifts to a subrepresentation of the regular representation of $G$; thus its regular representation must decom-

Received by the editors April 19, 1978 and, in revised form, October 11, 1978.

AMS (MOS) subject classifications (1970). Primary 43A65, 43A40; Secondary 22E35, $22 E 50$.

Key words and phrases. Induced representations, square-integrable representations, Plancherel measure, $p$-adic numbers. 
pose into a direct sum. In $\$ 3$ we sharpen this result by constructing a three-dimensional $p$-adic matrix group which is a Fell group and has a noncompact normal subgroup such that the quotient is not a Fell group. Then we generalize this result by exhibiting, for each dimension, an analogous example.

All the examples up to this point are nonalgebraic $p$-adic matrix groups. Nevertheless, it is possible to decompose each of them as the semidirect product of a reductive and a unipotent subgroup; this decomposition is analogous to the Levi decomposition for algebraic groups. All the examples of Fell groups constructed so far have the following feature in common: the reductive factor in this "Levi-type decomposition" is compact. In $\$ 4$ we show that this behavior is not a general phenomenon, by exhibiting, for each integer $n$, a Fell group of $n$-dimensional $p$-adic matrices whose Levi-type decomposition has a noncompact reductive factor. These examples also settle in the affirmative the question of the existence of nonamenable Fell groups. The fact that, from the measure-theoretical point of view, the dual objects of Fell groups and those of compact groups are very similar might suggest that Fell groups cannot contain infinite discrete subgroups. It is easy to check that this property holds in the case of Fell's example; however, the groups constructed in $\$ 4$ do not have this property. The discrete subgroups contained in them, however, are not normal. Finally in $\$ 5$, we exhibit a Fell group which is not a finite-dimensional matrix group and contains an infinite discrete normal subgroup.

All the examples of Fell groups we will exhibit consist of totally disconnected groups. It is reasonable to conjecture that, at least in the type I amenable case, no Lie group can be a Fell group: this conjecture is still unsettled in general, and a proof is only available under somewhat restrictive hypotheses.

The authors are indebted to Larry Corwin for helpful suggestions related to the examples in $\S 4$.

Unexplained terminology and notations are as in [3], [5].

2. Groups of rigid motions of $p$-adic spaces. In this and in the following sections several examples of Fell groups will be constructed. Most of these examples will be realizable as $p$-adic matrix groups. In all these cases, the analogous $p$-adic matrix groups over the corresponding local fields of characteristic $p$ will also be Fell groups. Noticing this fact once and for all, we will always restrict our attention to the case of characteristic zero.

Fell's example, which we will denote by $G_{1}$, consists of a unimodular solvable group, the semidirect product of the $p$-adic torus $\mathcal{T}_{p}$ and the additive group of the $p$-adic field $\mathscr{2}_{p}$, where the action is given by multiplication. In this section we will construct higher-dimensional generalizations of this example, which can be naturally regarded as the $p$-adic analogues of the Euclidean groups of real or complex spaces. This construction provides examples of nonsolvable Fell groups, and it also shows that the dimensions of the 
representations outside the discrete series of a Fell group may be unbounded; in the next section it will be shown that these dimensions may even be infinite.

We construct a family of Fell groups $G_{n}$ in the following way: denote by $\hat{\theta}_{p}$ the ring of integers in $\mathscr{2}_{p}$, and define $\operatorname{GL}\left(n, \mathcal{O}_{p}\right)$ as the group of $n \times n$ matrices with entries in $\vartheta_{p}$ and determinant of valuation one. Now define $G_{n}$ as the semidirect product

$$
G_{n}=\mathrm{GL}\left(n, \theta_{p}\right) \cdot 2_{p}^{n}
$$

where the action is the natural matrix action. Since $\operatorname{GL}\left(n, \theta_{p}\right)$ is compact, $G_{n}$ is a regular semidirect product; its irreducible unitary representations can be obtained via Mackey's little group theorem, once we describe the orbits of $\operatorname{GL}\left(n, \vartheta_{p}\right)$ over $\left(2_{p}^{n}\right)^{n}$. Since $2_{p}^{n}$ is self-dual, we have only to look at the orbits under the matrix action of $\operatorname{GL}\left(n, \theta_{p}\right)$ over $2_{p}^{n}$.

Let $\left(x_{1}, \ldots, x_{n}\right)$ be an element of $2_{p}^{n}$ : the $l^{\infty}$-sphere to which it belongs is defined as the set

$$
\left\{\left(w_{1}, \ldots, w_{n}\right) \in \mathcal{2}_{p}^{n}: \max _{i}\left|w_{i}\right|=\max _{i}\left|x_{i}\right|, i=1, \ldots, n\right\} .
$$

We claim that the nontrivial orbits of $\operatorname{GL}\left(n, \theta_{p}\right)$ over $2_{p}^{n}$ are $l^{\infty}$-spheres. $\operatorname{GL}\left(n, \theta_{p}\right)$ preserves the $l^{\infty}$-spheres since, if $T$ is in $\operatorname{GL}\left(n, \theta_{p}\right)$ and $T\left(x_{1}, \ldots, x_{n}\right)=\left(z_{1}, \ldots, z_{n}\right)$, it follows that

$$
\max _{i}\left|z_{i}\right|=\max _{i}\left|\sum_{j=1}^{n} t_{i j} x_{j}\right| \leqslant \max _{i}\left|x_{i}\right|
$$

because of the nonarchimedean property and the inequality $\left|t_{i j}\right| \leqslant 1$ for all $i$, $j$.

Furthermore, $\operatorname{GL}\left(n, \theta_{p}\right)$ acts transitively on these spheres, because if $\left(w_{1}, \ldots, w_{n}\right)$ is in $2_{p}^{n}$, there is always a $T$ in $\operatorname{GL}\left(n, \mathcal{\Theta}_{p}\right)$ such that

$$
\left(w_{1}, \ldots, w_{n}\right)=T(x, 0,0 \ldots 0)
$$

where $x$ is a $p$-adic number such that $|x|=\max _{i}\left|w_{i}\right|$. In fact, the above equality is equivalent to the following: $t_{i 1} x=w_{i}$ for $i=1, \ldots, n$; if $\left(w_{1}, \ldots, w_{n}\right)$ is not the zero element (corresponding to the trivial orbit), then set $t_{i 1}=x^{-1} w_{i}$ for all $i$. Since $\left|x^{-1} w_{i}\right| \leqslant 1, t_{i 1}$ belongs to $\theta_{p}$, and since $\max _{1<i<n}\left|x^{-1} w_{i}\right|=1$, it is always possible to complete the $n$-tuple $\left\{t_{i 1}\right\}$ to a matrix $T=\left\{t_{i j}, i, j=1, \ldots, n\right\}$ in $\operatorname{GL}\left(n, \Theta_{p}\right)$, thus proving the claim.

The relevant fact is that these orbits have positive Haar measure in $2_{p}^{n}$. Noticing that the stabilizer $H_{m}$ of the $m$ th $l^{\infty}$-sphere is closed in $\operatorname{GL}\left(n, \mathcal{\theta}_{p}\right)$, hence compact (in fact, nonabelian if $n>2$ ), and applying the decomposition theorem for group extensions [5], we conclude that the irreducible unitary representations of $G_{n}$ consist of the corresponding representations of the factor $\operatorname{GL}\left(n, \theta_{p}\right)$ lifted to the whole of $G_{n}$, and of the discrete series of square-inte- 
grable representations $\pi_{j, m}$, given by

$$
\pi_{j, m}=\underset{H_{m} \cdot 2_{p}^{n} \uparrow G_{n}}{\operatorname{Ind}} \sigma_{j} \otimes \chi_{m}
$$

where $\sigma_{j} \in \hat{H}_{m}$ and $\chi_{m}\left(x_{1}, \ldots, x_{n}\right)=\exp \left(2 \pi i p^{m} x_{1}\right)$.

Hence $G_{n}$ is a Fell group. It is also quite easy to check that there are sequences of formal degrees which vanish at infinity.

3. Quotients of Fell groups. As we already know, the quotient of a Fell group by a compact normal subgroup is again a Fell group. For the examples given in \$2, these quotients correspond to new Fell groups, whose regular representations contain an irreducible faithful subrepresentation $\pi$ which generates the regular representation by taking successive tensor product powers. In other words, up to multiplicity, $\rho \sim \bigoplus_{n>1} \pi^{n}$, where $\pi^{n}$ is the $n$th tensor power of $\pi$. For instance, let us consider the group $G_{1}$. Set $\pi_{m}=$ ind $_{2_{p} \uparrow G} \chi_{m}$ (notation as in $\$ 2$ ), denote by $A_{m}$ the subspace of the Fourier algebra $A\left(G_{1}\right)$ (see [3]) generated by the coefficients of $\pi_{m}$, and denote by $K_{j}$ the subgroup $\left\{\left(1, p^{j} \vartheta_{p}\right)\right\}$ of $G_{1}$. Let now $H_{j}=G_{1} / K_{j}$. Then the $K_{j}$ 's are the only compact normal subgroups of $G_{1}$, and, up to equivalence, the set of irreducible square-integrable representations of $G_{1}$ which are constant on the $K_{j}$-cosets is $\left\{\pi_{m}, m \leqslant j\right\}$. If $m \leqslant j$, denote by $\tilde{\pi}_{m}$ the quotient representation determined by $\pi_{m}$ on $H_{j}$ : since $\operatorname{ker} \pi_{j}=K_{j}$, $\tilde{\pi}_{j}$ is faithful on $H_{j}$; moreover, $\left\{\tilde{\pi}_{m}, m \leqslant j\right\}$ is, up to equivalence, the set of all irreducible square-integrable representations of $H_{j}$. For $m \leqslant j$, denote by $\tilde{A}_{m j}$ the image of $A_{m}$ under the canonical projection of $G_{1}$ onto $H_{j}$. It is easy to check, via the Mackey subgroup theorem, that $A_{m} \cdot A_{l} \simeq A_{l}$ if $l<m$, and that $A_{m} \cdot A_{m} \simeq A_{m} \oplus$ $A_{m-1}$; thus

$$
A\left(H_{j}\right) \simeq \bigoplus_{n<j} \tilde{A}_{n j} \simeq \bigoplus_{m>1}\left(\tilde{A}_{j j}\right)^{m}
$$

(where the $m$ th power stands for the subspace of $A\left(H_{j}\right)$ generated by the products of $m$ functions in $\tilde{A}_{j j}$ ). Equivalently, up to multiplicity, $\rho_{H_{j}} \sim$ $\bigoplus_{m>1} \tilde{\pi}_{j}^{m}$ (see [7] for details). Since the formal degree of $\pi_{m}$ decreases as $m \rightarrow-\infty$ [7], the product formula $A_{m} \cdot A_{l} \simeq A_{l}(l<m)$ implies that the formal degrees of the square-integrable irreducible representations of $G_{1}$ decrease under tensor product. Thus the property of $A\left(H_{j}\right)$ of being generated, as an algebra, by $\tilde{A}_{j}$, corresponds to the fact that the formal degrees of the square-integrable irreducible representations of $\boldsymbol{H}_{j}$ are bounded above (by the formal degree of the faithful representation $\tilde{\pi}_{j}$ ). A similar argument holds for the higher dimensional $G_{n}$, only slightly more complicated because of the existence of nontrivial stability subgroups. For a more general discussion of the relation between square-integrable irreducible faithful representations of a locally compact group $G$ and translation-invariant generating subspaces of $A(G)$, see [7, Theorem 1.2]. 
For all the previous examples it turns out that the quotients by noncompact normal subgroups also have purely atomic Plancherel measures (in fact, they are always compact). We construct here Fell groups with quotients whose Plancherel measure is not purely atomic. Let us first study a single example in detail.

Regarding Fell's example $G_{1}$ as the group of all $p$-adic matrices of the kind

$$
\left(\begin{array}{ll}
a & b \\
0 & a^{-1}
\end{array}\right), \quad a \in \mathcal{T}_{p}, \quad b \in \mathcal{Q}_{p},
$$

construct the semidirect product $H_{1}=G_{1} \cdot \mathscr{2}_{p}^{2}$, defined by the natural matrix action. $H_{1}$ is a regular semidirect product, and $G_{1}$ acts on $\left(\mathcal{Q}_{p}^{2}\right)^{\wedge} \simeq \mathscr{Q}_{p}^{2}$ by the rule

$$
(a, b) \cdot\left(\gamma_{1}, \gamma_{2}\right)=\left(a \gamma_{1}, b \gamma_{1}+a^{-1} \gamma_{2}\right)
$$

where $(a, b) \in G_{1},\left(\gamma_{1}, \gamma_{2}\right) \in\left(\mathcal{Q}_{p}^{2} \hat{)}\right.$.

Thus the orbits are the sets $\left(\mathscr{T}_{p} \cdot \gamma_{1}, \mathscr{Q}_{p}\right)$, if $\gamma_{1} \neq 0$, with trivial stabilizers, and the sets $\left(0, \mathcal{T}_{p} \cdot \gamma_{2}\right)$ if $\gamma_{1}=0, \gamma_{2} \neq 0$, with stabilizer $\left\{\left(\begin{array}{ll}1 & b \\ 0 & 1\end{array}\right), b \in \mathcal{Q}_{p}\right\}$; if $\gamma_{2}$ also is zero, the orbit is trivial and its stabilizer is the whole of $G_{1}$. The orbits corresponding to nonzero $\gamma_{1}$ 's have positive measure and give rise to a discrete series of representations; the complement of the union of these orbits is a null set in $2_{p}^{2}$. Therefore, by the decomposition theorem for Plancherel measures, $H_{1}$ is a Fell group. Now, the subgroup $N=\left\{(x, 0) \in \mathcal{Q}_{p}^{2}\right\}$ is normal in $H_{1}$ and noncompact, and the quotient $H_{1} / N$ is isomorphic to the direct product $G_{1} \times \mathcal{Q}_{p}$, which obviously is not a Fell group.

Notice also that each orbit containing an element of the kind $\left(0, \gamma_{2}\right) \in \mathcal{Q}_{p}^{2}$ gives rise to a series of infinite-dimensional representations which are not square-integrable.

The above construction readily generalizes to higher dimensions. To see this in a uniform fashion, observe that $G_{1}$ is isomorphic to the group of matrices $\left\{\left(\begin{array}{ll}a & b \\ 0 & 1\end{array}\right), a \in \mathcal{T}_{p}, b \in \mathcal{Q}_{p}\right\}$. Therefore $H_{1}$ is naturally isomorphic to the semidirect product of the $p$-adic torus $\mathcal{T}_{p}$ and the three-dimensional $p$-adic Heisenberg group $N_{1}$, identifying an element $t \in \mathcal{F}_{p}$ with the $3 \times 3 p$-adic matrix $\left\{t_{i j}\right\}$ defined by $t_{11}=t, t_{i j}=\delta_{i j}$ for $(i, j) \neq(1,1)$; the action of $\mathscr{T}_{p}$ over $N_{1}$ is now given by matrix multiplication. Now, just as above, one can identify $\mathcal{T}_{p}$ with a group of $n \times n$ matrices and construct the semidirect product $H_{m}=\mathcal{T}_{p} \cdot N_{m}$, where $N_{m}$ is the $p$-adic Heisenberg group of dimension $2 m+1$, and the action is given by matrix multiplication. By the same argument as before, $H_{m}$ is a Fell group. The subgroup $N$ of $H_{1}$ defined above is nothing else but the center of the unipotent factor $N_{1}$. In the case of $H_{m}$, the center $Z$ of the unipotent factor $N_{m}$, being a characteristic subgroup of $N_{m}$, is nonnal in $H_{m}$, and again it turns out that the quotient $H_{m} / Z$ is not a Fell group, because it contains an abelian direct factor. In higher dimensions, one may form other quotients of $H_{m}$ which are not Fell groups; we omit the details here. 
4. A family of nonamenable Fell groups. In the last section, we proved that quotients of Fell groups are not necessarily Fell groups. All the groups constructed so far are not algebraic. Nevertheless they admit a decomposition as semidirect products of a reductive and a unipotent factor, analogous to the Levi decomposition for algebraic groups; groups of this kind will be called almost-algebraic. In all the previous examples, the reductive factors turn out to be compact; in other words, the quotients of these Fell groups by their unipotent "Levi" factors do have purely atomic Plancherel measures. In the present section, we will construct an example of an almost-algebraic Fell group whose reductive Levi factor is noncompact.

In particular, this construction yields examples of nonamenable Fell groups.

Let $U L\left(n, 2_{p}\right)$ be the group of $n \times n p$-adic matrices with determinant of valuation 1. Denote by $K_{n}$ the semidirect product of $U L\left(n, \mathscr{2}_{p}\right)$ and the additive group $M\left(n, \mathscr{Q}_{p}\right)$ of all $n \times n p$-adic matrices, where the action is given by matrix multiplication. Notice that $K_{n}$ is unimodular and $M\left(n, \mathscr{Q}_{p}\right)$ is isomorphic to the vector space $2_{p}^{n^{2}}$, which is self-dual. The subgroup of $M\left(n, \mathcal{Q}_{p}\right)$ consisting of matrices with determinant zero is a null set in $2_{p}^{n^{2}}$; on the other hand, if an element of $M\left(n, \mathscr{Q}_{p}\right)$ has determinant $\alpha \neq 0$, its orbit under $U L\left(n, 2_{p}\right)$ consists of all the matrices whose determinant has valuation $|\alpha|_{p}$. Therefore, up to a null set, $M\left(n, \mathscr{Q}_{p}\right)$ is the union of (countably many) orbits of positive measure, and it is obvious that the stabilizers of these orbits are trivial: hence $K_{n}$ is a Fell group.

It is clear that $K_{n}$ is almost-algebraic: it can be realized as the group of $p$-adic matrices of dimension $n^{2}+1$ of the kind $T_{A}+M_{B}$, where $T_{A}$ and $M_{B}$ are defined as follows.

The only nonzero coefficients of $T_{A}$ are given by the rules

$$
\begin{aligned}
& \left(T_{A}\right)_{n^{2}+1, n^{2}+1}=1, \\
& \left(T_{A}\right)_{k n+i, k n+j}=A_{i j}, \quad 0 \leqslant k<n, \quad 1 \leqslant i \leqslant n, \quad 1 \leqslant j \leqslant n .
\end{aligned}
$$

Finally, for every $B$ in $M\left(n, \mathscr{Q}_{p}\right), M_{B}=\left\{m_{i j}\right\}$ is the matrix of dimension $n^{2}+1$ whose only nonzero entries are given by $m_{r, n^{2}+1}=b_{j, k}$, where $r=j+$ $(k-1) n$.

The groups $K_{n}$ are also nonamenable, because the factor $U L\left(n, \mathcal{Q}_{p}\right)$ is the semidirect product of the $p$-adic torus $\mathcal{T}_{p}$ and the unimodular $p$-adic group $\operatorname{SL}\left(n, \mathscr{2}_{p}\right)$, a semisimple group which is known to be not amenable: the argument that shows that semisimple Lie groups with compact center are nonamenable (a comparison between modular functions: see [4, p. 18]) also holds for $\operatorname{SL}\left(n, \mathcal{Q}_{p}\right)$, via [2, Lemma 1]. The compact open subgroups form a partially ordered set whose inductive limit is the proper subgroup $\operatorname{GL}\left(n, \theta_{p}\right)$. $M\left(n, \mathscr{Q}_{p}\right)$. Finally, it is clear that the reductive Levi factor of $K_{n}$ is the noncompact group $U L\left(n, \mathscr{Q}_{p}\right)$.

We also notice the following feature of the groups $K_{n}$ : they are Fell groups which contain infinite discrete subgroups. Because of the similarity between 
compact and Fell groups (at least as far as their representation theory is concerned), one might have expected that Fell groups do not contain infinite discrete subgroups. Indeed this property is easily verified for all the previous examples; it does not hold, however, for the groups exhibited in the present section, since one can pick infinite discrete subgroups in $U L\left(n, \mathscr{Q}_{p}\right)$, hence in $K_{n}$. Moreover, these subgroups can be chosen torsion-free. It is easy to select, for instance, a discrete subgroup of $\operatorname{SL}\left(n, \mathscr{2}_{p}\right)$-hence of $U L\left(n, \mathscr{Q}_{p}\right)$-which is isomorphic to the integers. Noncommutative examples can also be produced: we will now show that $\operatorname{SL}\left(n, \mathscr{Q}_{p}\right)$ contains a discrete subgroup isomorphic to the free group on two generators. It is enough to show this for $n=2$, since $\operatorname{SL}\left(2, \mathscr{Q}_{p}\right)$ is naturally isomorphic to a closed subgroup of $\operatorname{SL}\left(n, 2_{p}\right)$. Set

$$
P=\left(\begin{array}{ll}
p & 1 \\
0 & p^{-1}
\end{array}\right), \quad R=\left(\begin{array}{rr}
0 & 1 \\
-1 & 0
\end{array}\right),
$$

and consider the subgroup $D$ of $\operatorname{SL}\left(2, \mathscr{2}_{p}\right)$ generated by $P$ and $T=R P R$. We claim that $D$ is isomorphic to the free group on two generators. Indeed, suppose the elements $T$ and $P$ satisfy some relations: since $R$ is an involution, this is equivalent to saying that there exist integers $n_{1} \ldots n_{k}$ such that $R^{e} P^{n_{1}} R P^{n_{2}} \ldots R P^{n_{k}}=1$, where 1 is the identity element and $\varepsilon=0$ or 1 . If $n>0$, set

$$
q_{n}=\sum_{k=0}^{n-1} p^{1-n+2 k}
$$

and notice that

$$
P^{n}=\left(\begin{array}{ll}
p^{n} & q_{n} \\
0 & p^{-n}
\end{array}\right)=\left(\begin{array}{ll}
\alpha & \beta \\
0 & \alpha^{-1}
\end{array}\right),
$$

with $|\alpha|_{p}=p^{n},|\beta|_{p}=p^{n-1}$. Then

$$
R P^{n}=\left(\begin{array}{rr}
-\alpha^{-1} & 0 \\
\beta & -\alpha
\end{array}\right),
$$

and by the nonarchimedean property of the $p$-adic valuation one can easily show that the lower left entry of the matrix $R P^{n_{1}} \ldots R P^{n_{k}}$ has valuation $p^{\left(\Sigma n_{i}\right)-k}$, if $n_{i}>0,1 \leqslant i \leqslant k$; in particular, this entry is nonzero, while the upper right entry is zero. The same is true if some of the $n_{i}$ are negative. Therefore $R^{\varepsilon} P^{n_{1}} R P^{n_{2}} \ldots R P^{n_{k}} \neq 1$, so that $D$ is a free group. A similar estimate of the valuation of the entries of the elements of $D$ shows that $D$ is discrete.

We have selected various kinds of discrete torsion-free subgroups of $K_{n}$. Notice that no such subgroup is normal. In the following section we will construct an example of a Fell group with an infinite discrete normal subgroup; this group, however, will not be a $p$-adic matrix group, and the discrete subgroups will not have torsion-free elements. 
5. A Fell group which is not a $p$-adic matrix group. To complete our constructions, in this section we enlarge the natural environment in which Fell groups have been constructed so far, and exhibit a Fell group which is not almost algebraic: it still consists of a matrix group, but its elements are infinite-dimensional matrices over a discrete field. This example was first studied in [6], in a slightly different context.

Denote by $\mathrm{Z}_{2}$ the field with two elements, and let $N$ be the infinite-dimensional vector space $\Sigma^{\oplus} \mathbf{Z}_{2}$, where the sum is countable; endowed with the discrete topology, $N$ is a discrete abelian group: its dual is the Cantor group D. Now, let $K$ be the group of all automorphisms of the vector space $N$ which, in the canonical basis for $N$, correspond to upper triangular matrices. $K$ can be imbedded, as a closed subset, into the product of infinitely many copies of the compact space $\mathbf{Z}_{2}$, and therefore it is compact. Define the semidirect product $G=K \cdot N$, where the action is the natural matrix action; since $K$ is compact, $N$ is regularly embedded in $G$, and Mackey's theory applies.

Denote by $\mathbf{e}=\left\{e_{i}, i=1,2, \ldots\right\}$ an element of $\mathbf{D}=\Pi \mathbf{Z}_{2}$. We have to look at the action of $K$ on $\mathbf{D}$; a trivial computation shows that, for $k \in K$, this action is given by $k \cdot \mathbf{e}=\left(k^{-1}\right)^{t} \mathbf{e}$.

Therefore one obtains a countable family of orbits $\vartheta_{i}, i=1,2, \ldots$, defined by

$$
\vartheta_{i}=\left\{\mathbf{e} \in \mathbf{D}: e_{j}=0 \forall j<i, e_{i}=1\right\}
$$

together with the trivial orbit $\theta_{\infty}=\{0\}$.

Since the orbit $\vartheta_{i}$ has Plancherel measure $2^{-i}$, and its stabilizer is compact, $G$ is a Fell group. Notice that, as mentioned before, $G$ contains the infinite discrete abelian normal subgroup $N$ : however, every nontrivial element in $N$ has order 2.

\section{BIBLIOGRAPHY}

1. L. Bagget, A separable group having a discrete dual space is compact, J. Functional Analysis, 10 (1972), 131-148.

2. F. Bruhat, Sur les représentations des groupes classiques p-adiques. I, Amer. J. Math. 83 (1961), 321-338.

3. P. Eymard, L'algèbre de Fourier d'un groupe localement compact, Bull. Soc. Math. France 92 (1964), 181-236.

4. __ Moyennes invariantes et représentations unitaires, Lecture Notes in Math., vol. 300, Springer-Verlag, Berlin, 1972.

5. A Kleppner and R. L. Lipsman, The Plancherel formula for group extensions, Ann. Sci. École Norm. Sup. 5 (1972), 71-120.

6. J. R. Liukkonen and M. W. Mislove, Symmetry in Fourier-Stieltjes algebras, Math. Ann. 217 (1975), 97-112.

7. G. Mauceri, Square-integrable representations and the Fourier algebra of a unimodular group, Pacific J. Math. 73 (1977), 143-154.

8. N. W. Rickert, Convolution of $L^{2}$ functions, Colloq. Math. 19 (1968), 301-303.

Department of Mathematics, University of Genoa, 16100 Genoa, Italy

Department of Mathematics, University of Rome, 00100 Rome, ItAly 